\title{
A Fair Pricing Approach to Weather Derivatives
}

\author{
Eckhard Platen $^{1}$ and Jason West ${ }^{2}$
}

November 1, 2004

\begin{abstract}
This paper proposes a consistent approach to the pricing of weather derivatives. Since weather derivatives are traded in an incomplete market setting, standard hedging based pricing methods cannot be applied. The growth optimal portfolio, which is interpreted as a world stock index, is used as a benchmark or numeraire such that all benchmarked derivative price processes are martingales. No measure transformation is needed for the proposed fair pricing. For weather derivative payoffs that are independent of the value of the growth optimal portfolio, it is shown that the classical actuarial pricing methodology is a particular case of the fair pricing concept. A discrete time model is constructed to approximate historical weather characteristics. The fair prices of some particular weather derivatives are derived using historical and Gaussian residuals. The question of weather risk as diversifiable risk is also discussed.
\end{abstract}

1991 Mathematics Subject Classification: primary 90A12; secondary 60G30, 62P20. JEL Classification: C16, G10, G13

Key words and phrases: weather derivatives, benchmark approach, growth optimal portfolio, fair pricing, actuarial pricing.

\footnotetext{
${ }^{1}$ University of Technology Sydney, School of Finance \& Economics and Department of Mathematical Sciences, PO Box 123, Broadway, NSW, 2007, Australia

${ }^{2}$ Corresponding author. Jason West, School of Finance and Economics, University of Technology, Sydney, P.O. Box 123, Broadway NSW 2007, Australia; Tel. +61 29514 7772; fax: +61 29514 7711; e-mail: jason.m.west@uts.edu.au.
} 


\section{Introduction}

The impact of weather on many commercial and recreational activities is significant and varies both geographically and seasonally. Many businesses, including agriculture, insurance, energy and tourism, are either favorably or adversely affected by weather. For this reason, the financial markets have devised a relatively new class of instruments, called weather derivatives, through which risk exposure to weather may be transferred or reduced. Weather derivatives are contingent claims written on weather indices, which in turn are variables whose values are constructed from weather data. Commonly referenced weather indices include, but are not restricted to, daily average temperature (DAT), cumulative annual temperature (CAT), heating degree days (HDDs), cooling degree days (CDDs), precipitation, snowfall and wind. The flexibility of defining a specific weather index and writing a contingent claim against it allows innovative hedging structures to be developed. This allows the management of an almost unlimited variety of weather-related risks. An interesting example of applying weather risk swaps is given in Kariya (2003). In addition, it is widely perceived that the correlation between weather indices and most established financial indices is negligible. The ability of financial analysts to price these instruments is, so far, somewhat restricted. The traditional hedging based pricing methodology does not provide a practical pricing method for valuing weather derivatives, because the underlying weather indices are at present still not securitized by liquidly traded instruments.

There also exist some difficulties in implementing statistical, equilibrium based pricing techniques, because the observed weather indices are non-stationary. They are characterized by long-term variations and trends, potentially with cycles much longer than what the weather data records reveal. It is a straightforward observation that the actuarial present value pricing approach is rather simple and intuitively appealing. It would be convenient if one could apply this pricing methodology to weather derivatives on the basis of a firm theoretical argument. Furthermore, linking weather derivative pricing to an intuitively appealing actuarial type problem would be ideal for the evaluation of exotic weather derivative instruments with complex payoff structures. This paper will provide such a basis for the pricing of weather derivatives. It exploits a deep link between financial and actuarial pricing. The approach still works when an equivalent risk neutral martingale measure does not exist.

The fair pricing concept outlined below will allow us to consistently price any financial and weather instrument. It is part of the benchmark approach derived in Platen (2002) and Bühlmann \& Platen (2003) and exploits the notion of the growth optimal portfolio (GOP), originally developed by Kelly (1956). The GOP is the self-financing portfolio that maximizes expected logarithmic utility from terminal wealth. It was later extended and applied, for instance, by Long (1990), BajeuxBesnainou \& Portait (1997), Platen (2002), Goll \& Kallsen (2003) and Bühlmann \& Platen (2003). 
Under certain conditions, the GOP coincides with the numeraire portfolio, see Long (1990), Becherer (2001) and Platen (2004b), which converts prices, when expressed in units of the numeraire, into martingales under the historical or real world probability measure. The practice of expressing prices in terms of the GOP is called benchmarking. In a semimartingale setting, Platen (2004a) demonstrates that when prices are benchmarked, they become supermartingales without any major assumptions being imposed on the model.

For simplicity and for practical purposes, we will follow the discrete time benchmark approach here, see Bühlmann \& Platen (2003). A derivative price is said to be fair if its benchmarked value follows a martingale. Thus, the key to fair pricing under the benchmark approach requires only the computation of expected benchmarked values. From this, the fair price of a weather derivative can be obtained in a consistent manner via conditional expectations with respect to the real world probability measure. We will see that since weather related payoffs can be assumed to be independent of the GOP, this then recovers the standard actuarial pricing method. Fair prices in relation to weather related instruments can thus be obtained by present value pricing, even for models where no equivalent risk neutral martingale measure exists. The question of weather risk as diversifiable risk is also discussed.

In an illustration towards the end of this paper, at first, predictable seasonalities and trends in the modeling of weather indices are estimated for a given locality. The available historical data allows us then to obtain a substantial record of resulting random residuals. Under the fair pricing concept, the historical fair pricing (HFP) method is then used and justified, via the Law of Large Numbers. The HFP computes an estimated expected future payoff of a weather derivative using all historical data. The available recorded temperature data for Sydney will be used to illustrate the HFP method for determining certain weather derivative prices.

It will be shown that the Central Limit Theorem can be exploited to support some Gaussianity property of certain weather indices, which are formed by sufficiently large samples of reasonably independent contributions to their values. Thus, the assumption of normality can be justified for certain weather indices. This distributional property can be then exploited for the computation of expected weather derivative payoffs.

The paper is organized as follows: Section 2 outlines various pricing approaches, including the benchmark approach using the GOP. It also establishes the link between fair and actuarial pricing. Section 3 introduces the historical weather data sets used to examine the past behavior of weather indices and represents some modeling. Section 4 applies the HFP method and calculates prices for particular weather derivatives based on historical residuals. For illustration, it also derives weather derivative prices for a particular agricultural example. 


\section{Pricing Approaches}

The emergence of innovative products, which aim to securitize the core risks to a particular industry, has led researchers to question the validity of existing pricing methods that are either based on hedging arguments, see Black \& Scholes (1973), or a belief in the Law of Large Numbers, see Borch (1968). Insurance and weather derivatives, catastrophe bonds and other contingent claim securities, typically placed in incomplete markets, have challenged the traditional no-arbitrage and actuarial pricing methods used in finance and insurance, respectively.

The pricing of derivatives in incomplete markets, see Hofmann, Platen \& Schweizer (1992) and Heath, Platen \& Schweizer (2001), generally leads to pricing concepts based on certain expected value calculations. Until the early 1990s, there had been relatively little research into merging the actuarial and no-arbitrage pricing methodologies, with formal mathematical rigor.

Several attempts have been made to price weather derivatives using the traditional financial or no-arbitrage pricing approach, for example McIntyre \& Doherty (1999) and Leggio \& Lien (2002). However, this does not yield robust results, since the underlying security or index is not tradable, and it is not clear what market price for risk should be chosen. With special reference to this last point, it has been claimed by Cao \& Wei (2001) that the risk premium in the value of a weather derivative security is zero if the process governing dividends on the market portfolio is completely independent of the weather index process. Therefore, they conclude that any contingent claim can be valued by discounting its payoff at the risk-free rate. We will provide some mathematically founded arguments that support such a pricing rule for liquid, competitive markets.

The benchmark approach, proposed in Platen (2002), generalizes various pricing methods through the use of the growth optimal portfolio (GOP) as benchmark. For general benchmark models benchmarked nonnegative portfolios are supermartingales, see Platen (2004a). A particular situation arises if a benchmarked price process is a martingale, then this price process is called fair. The benchmark approach also covers models where an equivalent risk neutral martingale measure does not exist. As shown in Platen (2004b), this situation is likely for realistic market models. In the case when an equivalent risk neutral martingale measure exists, the GOP acts as numeraire portfolio, see Long (1990), from which the fair price of a particular derivative instrument can be derived by taking conditional expectations under the real world probability measure. In this case this price can be shown to coincide with the standard risk neutral price. Under more general assumptions we will show, under the benchmark approach, that a generalized actuarial price is obtained as the fair derivative price when the payoff is independent of the GOP. The benchmark approach will therefore lead to a similar, but more detailed conclusion as Cao \& Wei (2001), using a mathematically rigorous framework. It will be emphasized that in the case of independence between the GOP and the underlying weather index the benchmarked fair price decouples, due to 
the martingale property of benchmarked fair prices into the expected payoff and a zero coupon bond price as discounted factor. This yields the generalized actuarial pricing formula. Furthermore, the assumed observed independence of weather indices and the GOP can be interpreted to be equivalent to the absence of weather risk premia, which means that the market price for weather risk is zero. This is consistent with the common observation that weather risk is geographically and temporally diversifiable. However, in reality, in the still emerging weather market traded weather derivative prices may attract some positive liquidity premia and hence their traded prices may exceed their fair prices. As the weather derivative market develops liquidity premia can be expected to vanish.

\subsection{Actuarial and Risk Neutral Pricing}

In what follows in this subsection, different valuation methods will be discussed in basic terms, followed by a comprehensive outline of the discrete time benchmark approach in subsequent sections including a more detailed analysis of actuarial and risk neutral pricing. For simplicity, let us assume that the short rate $r$ is a deterministic constant. Taking at the initial time $t=0$ the expectation

$$
C_{H_{T}}(0)=e^{-r T} E\left(H_{T}\right)
$$

of a discounted cashflow $H_{T}$ at time $T$, leads us at time $t=0$ to the actuarial price of that cashflow. Note that the expectation $E(\cdot)$ is here taken with respect to the real world probability measure $P$. For instance, in insurance, on top of this value, a premium is sometimes charged to the insured if possible. In a competitive economy, market forces put pressure on insurers to reduce such premiums, in particular, when reinsurance is available. We are not considering these insurance approaches any further. The reader is referred to Borch (1968) for a detailed description of insurance pricing concepts. In weather derivative pricing a liquidity premium is likely to be charged by sellers of these derivatives as long as the market accepts that. Based upon the benchmark approach and its concept of fair pricing the present paper endorses the view of Cao \& Wei (2001) who claim that there is no weather risk premium. This is also supported by the argument that weather risk is diversifiable in the global world market. We emphasize that fair pricing assumes in practice a competitive and liquid market.

It is well-known that if there exists, in a complete financial market context, an equivalent risk neutral martingale measure $Q$, then the price of any instrument in this market can be expressed as an expected value with respect to this measure. The risk neutral price $C_{H_{T}}^{Q}(0)$ at the initial time $t=0$ of the payoff $H_{T}$ at time $T$ is then

$$
C_{H_{T}}^{Q}(0)=e^{-r T} E^{Q}\left(H_{T}\right) .
$$

Here $E^{Q}(\cdot)$ denotes expectation with respect to $Q$. More generally, under the benchmark approach, see Platen (2002), in a continuous complete market model any contingent claim can be hedged with a self-financing portfolio. The fair price 
then represents the value of a corresponding hedge portfolio. If an equivalent risk neutral martingale measure does not exist, then it can be shown that the fair price provides the minimal hedge portfolio, as described in Platen (2002). If the market is incomplete, only bounds on arbitrage free prices may be derived, see Platen (2004c). This creates an unsatisfactory situation, in particular, for weather derivative pricing, where the market must be considered to be incomplete at present. In Platen (2004a) the benchmark approach has been established that allows us to deal with this situation and will be described in the following.

\subsection{Benchmark Approach}

\section{Discrete Time Model}

For simplicity, we consider a discrete time market, formulated on a given filtered probability space $\left(\Omega, \mathcal{A}_{T}, \underline{\mathcal{A}}, P\right)$, see Protter (1990). Asset prices are assumed to change their values only at the given discrete times

$$
0 \leq t_{0}<t_{1}<\ldots<t_{n}=T<\infty
$$

for $n \in\{1,2, \ldots\}$. The information structure in this market is described by the discrete filtration $\underline{\mathcal{A}}=\left(\mathcal{A}_{t_{i}}\right)_{i \in\{0,1, \ldots, n\}}$. Here $\mathcal{A}_{t}$ expresses the information available at time $t$.

Denote by $S_{i}^{(j)}$ the non-negative value at time $t_{i}$ of the $j$ th primary security account, which holds units and accumulated earnings of the $j$ th asset, $j \in\{0,1, \ldots, d\}$. Therefore, the $j$ th primary security account expresses the investment value, including the accumulation of earnings, of the $j$ th primary security. The 0th primary security account process $S^{(0)}=\left\{S_{i}^{(0)}, i \in\{0,1, \ldots, n\}\right\}$ is the domestic savings account. In the case where the underlying security is a stock the quantity $S_{i}^{(j)}$ represents the $j$ th cum-dividend share price at time $t_{i}$. It is assumed that the market observes its value at any given time. In addition, we assume that the initial value $S_{0}^{(j)}>0$ is positive for $j \in\{0,1, \ldots, d\}$.

The price ratio $h_{i}^{(j)}$ of the $j$ th primary security account at time $t_{i}$ is denoted by

$$
h_{i}^{(j)}=\left\{\begin{array}{cc}
\frac{S_{i}^{(j)}}{S_{i-1}^{(j)}} & \text { for } S_{i-1}^{(j)}>0 \\
0 & \text { otherwise }
\end{array}\right.
$$

for $i \in\{1,2, \ldots, n\}$ and $j \in\{0,1, \ldots, d\}$. The return of $S^{(j)}$ at time $t_{i}$ equals $h_{i}^{(j)}-1$. We can express the price of the $j$ th primary security account at time $t_{i}$ as

$$
S_{i}^{(j)}=S_{0}^{(j)} \prod_{m=1}^{i} h_{m}^{(j)},
$$

for $i \in\{1,2, \ldots, N\}$ and $j \in\{0,1, \ldots, d\}$. 
This leads us to the formation of a self-financing portfolio $S^{(\pi)}=\left\{S_{i}^{(\pi)}, i \in\right.$ $\{0,1, \ldots, n\}\}$, as defined in Bühlmann \& Platen (2003), where all changes in the value of the portfolio are due to changes in the values of the primary security accounts. Under a self-financing strategy no outflow or inflow of funds occurs for the corresponding portfolio. It is characterized by a vector process of fractions or proportions $\pi=\left\{\pi_{i}=\left(\pi_{i}^{(1)}, \ldots, \pi_{i}^{(d)}\right), i \in\{0,1, \ldots, n\}\right\}$ invested in the different primary security accounts. Here $\pi_{i}^{(j)}$ represents the proportion of the portfolio value held in the $j$ th individual primary security account, $j \in\{0,1, \ldots, d\}$. This proportion is observable at time $t_{i}$, that is $\mathcal{A}_{t_{i}}$-measurable. Furthermore, all proportions add up to one such that

$$
\sum_{j=0}^{d} \pi_{i}^{(j)}=1
$$

for all $i \in\{0,1, \ldots, n\}$. It follows for a strictly positive portfolio process $S^{(\pi)}=$ $\left\{S_{i}^{(\pi)}, i \in\{0,1, \ldots, n\}\right\}$ its price ratio

$$
h_{m}^{(\pi)}=\frac{S_{m}^{(\pi)}}{S_{m-1}^{(\pi)}}
$$

at time $t_{m}$ in the form

$$
h_{m}^{(\pi)}=\sum_{j=0}^{d} \pi_{m-1}^{(j)} h_{m}^{(j)}
$$

for $m \in\{1,2, \ldots, N\}$. By using the price ratios $h_{m}^{(\pi)}$, see (2.6), the portfolio value at time $t_{i}$ can be expressed as

$$
S_{i}^{(\pi)}=S_{0}^{(\pi)} \prod_{m=1}^{i} h_{m}^{(\pi)}
$$

for $i \in\{1,2, \ldots, n\}$. Note that the return of $S^{(\pi)}$ at time $t_{i}$ equals $h_{m}^{(\pi)}-1$.

Let us denote by $\mathcal{V}$ the class of all strictly positive self-financing portfolio processes $S^{(\pi)}$ such that $S_{0}^{(\pi)}=1$. The growth rate $g_{i}^{(\pi)}$ at time $t_{i}$ for a given portfolio process $S^{(\pi)} \in \mathcal{V}$, with process of proportions $\pi$, is defined as

$$
g_{i}^{(\pi)}=E\left(\ln \left(h_{i+1}^{(\pi)}\right) \mid \mathcal{A}_{t_{i}}\right)
$$

for all $i \in\{0,1, \ldots, n-1\}$. This expression provides a measure for the expected growth of wealth over the period $\left[t_{i}, t_{i+1}\right]$.

\section{Growth Optimal Portfolio}

Using equation (2.9), one can define the optimal growth rate $\underline{g_{i}}$ at time $t_{i}$ as the essential supremum

$$
\underline{g_{i}}=\operatorname{ess} \sup _{S^{(\pi)} \in \mathcal{V}} g_{i}^{(\pi)}
$$


over all strictly positive portfolios, for all $i \in\{0,1, \ldots, n-1\}$. Assume that a portfolio $S^{(\underline{\pi})} \in \mathcal{V}$ exists, where the associated process of proportions $\underline{\pi}$ results in

$$
g_{i}^{(\underline{\pi})}=\underline{g_{i}}<\infty
$$

and the expected ratio of price ratios

$$
E\left(\frac{h_{i+1}^{(\pi)}}{h_{i+1}^{(\underline{\pi})}} \mid \mathcal{A}_{t_{i}}\right)<\infty
$$

are finite for all $i \in\{0,1, \ldots, n-1\}$ and all proportions $\pi$ which lead to positive self-financing portfolios. The above portfolio $S^{(\underline{\pi})}$, for which we assumed its existence, is called a growth optimal portfolio (GOP). The condition (2.12) ensures that the GOP does not have an infinite expected growth rate. This definition of a GOP is rather general and covers, in principle, all practically relevant discrete time models, see Bühlmann \& Platen (2003), Goll \& Kallsen (2003) and Platen (2004a). Note that we did not specify any particular dynamics of the primary security accounts. For any model or model class this needs to be done and one has then to confirm that the properties (2.11) and (2.12) are satisfied.

Using (2.8) one can show that a GOP with associated proportions $\underline{\pi}$ is a portfolio that maximizes the expected value of the logarithm of the associated value process for every future time instant. Note that the value process of the GOP is unique. However, the process of proportions $\underline{\pi}$ may not be unique, in particular, for a market with redundant primary security accounts. For a detailed examination of the GOP, the reader is referred to, for instance, Korn \& Schäl (1999) and Fama \& MacBeth (1974).

Becherer (2001), Bühlmann \& Platen (2003) and Platen (2004a) prove under general assumptions that a strictly positive portfolio process $S^{(\underline{\pi})}$ is a GOP if and only if all nonnegative portfolios $S^{(\pi)} \in \mathcal{V}$, when expressed in units of $S^{(\underline{\pi})}$, are $(\underline{\mathcal{A}}, P)$-supermartingales, that is

$$
E\left(\frac{h_{i+1}^{(\pi)}}{h_{i+1}^{(\underline{\pi})}} \mid \mathcal{A}_{t_{i}}\right) \leq 1
$$

for all $i \in\{0,1, \ldots, n-1\}$. For instance, the benchmarked savings account seems, in reality, to behave like a strict supermartingale, see Platen (2004b). Note that Long (1990), in a discrete time model, showed under the existence of an equivalent risk neutral martingale measure that all benchmarked securities in a market become martingales. The result in (2.13) is more general and covers further important cases, where benchmarked nonnegative securities are permitted to be strict supermartingales. If there does not exists an equivalent risk neutral martingale measure, then there may exist arbitrage opportunities in the sense of a "free lunch with vanishing risk", see Delbaen \& Schachermayer (1994). However, due to the supermartingale property of benchmarked nonnegative portfolio processes there is 
no opportunity to generate strictly positive wealth from zero initial capital, see Platen (2002).

The GOP can be interpreted as a diversified portfolio, representative of the best performing maximization policy for the expected logarithm of terminal wealth. In the very long term, the growth optimal policy almost surely provides higher wealth and thus higher realized utility than alternative strategies, see Platen (2004a). Although, as already pointed out by Samuelson (1971), this does not imply that the growth optimal policy maximizes expected utilities for other utility functions.

It has been shown in Platen (2004b) that a diversified world stock accumulation index approximates the GOP under quite realistic assumptions. It is therefore appropriate to assume later on that the dynamics of the GOP is closely approximated by the MCSI World Index (MSCI). It is not surprising that it will turn out that the MSCI is practically independent of the dynamics of weather indices. This means, in practice, one can assume that the GOP is independent of any particular weather risk. This will become an important simplifying assumption. In what follows it will be shown that detailed specific knowledge of the GOP is not necessary for the proposed fair pricing of contingent claims of weather derivatives. Only its existence will be used to obtain a valid pricing formula. Furthermore, it will turn out that the interest rate term structure can be stochastic under the pricing formula that we will derive.

\section{Fair Pricing of Contingent Claims}

The benchmarked price $\hat{S}_{i}^{(\pi)}$ at time $t_{i}$ of a self-financing portfolio $S^{(\pi)} \in \mathcal{V}$ is defined by the ratio

$$
\hat{S}_{i}^{(\pi)}=\frac{S_{i}^{(\pi)}}{S_{i}^{(\underline{\pi})}}
$$

for all $i \in\{0,1, \ldots, n\}$, where the GOP $S_{i}^{(\underline{\pi})}$ is the numeraire. According to (2.13), the benchmarked price of a nonnegative self-financing portfolio $S^{(\pi)}$ is always a supermartingale, that is,

$$
\hat{S}_{i}^{(\pi)} \geq E\left(\hat{S}_{k}^{(\pi)} \mid \mathcal{A}_{t_{i}}\right)
$$

for all times $t_{i} \leq t_{k}$, where $i, k \in\{0,1, \ldots, n\}$.

We say that a price process $u=\left\{u_{i}, i \in\{0,1, \ldots, n\}\right\}$ is fair if its benchmarked value process $\hat{u}=\left\{\hat{u}_{i}=\frac{u_{i}}{S_{i}^{(\underline{\underline{\underline{\mu}}})}}, i \in\{0,1, \ldots, n\}\right\}$ is an $(\underline{\mathcal{A}}, P)$-martingale, that is

$$
\hat{u}_{i}=E\left(\hat{u}_{k} \mid \mathcal{A}_{t_{i}}\right)
$$

for all times $t_{i} \leq t_{k}$ with $i, k \in\{0,1, \ldots, n\}$.

To provide an example, consider the payoff function $H_{t_{n}}$ for a European option written on an underlying security $S_{n}$ with strike price $K$ and maturity $t_{n}$. For the European call option, $H_{t_{n}}$ is given as

$$
H_{t_{n}}=\left(S_{n}-K\right)^{+}=\max \left(S_{n}-K, 0\right) .
$$


From equation (2.17), the benchmarked payoff at maturity $t_{n}$ is

$$
\hat{H}_{t_{n}}=\frac{H_{t_{n}}}{S_{n}^{(\underline{\alpha})}}
$$

which represents the payoff expressed in units of the GOP at time $t_{n}$.

In order to determine the fair value $C_{H_{t_{n}}}\left(t_{i}\right)$ of the European option at time $t_{i} \leq t_{n}$, one first needs to compute, by formula (2.16), its benchmarked fair value $\hat{C}_{H_{t_{n}}}\left(t_{i}\right)$ as the conditional expectation

$$
\hat{C}_{H_{t_{n}}}\left(t_{i}\right)=E\left(\hat{H}_{t_{n}} \mid \mathcal{A}_{t_{i}}\right)
$$

By multiplication with the actual value $S_{i}^{(\underline{\pi})}$ of the GOP on both sides of (2.19), this yields then, in domestic currency, the fair pricing formula

$$
C_{H_{t_{n}}}\left(t_{i}\right)=S_{i}^{(\underline{\pi})} \hat{C}_{H_{t_{n}}}\left(t_{i}\right)
$$

for all $i \in\{0,1, \ldots, n\}$. The calculation of the fair price of a contingent claim is thus reduced to calculating an expectation of a benchmarked future payoff under the real world probability measure $P$. We emphasize that interest rates can be stochastic under fair pricing and completeness of the market is not required. Since there is no measure transformation involved in the fair pricing concept, an equivalent risk neutral martingale measure need not exist for whatever market model one chooses, assuming (2.11) and (2.12). Furthermore, we show below that standard risk neutral pricing and actuarial pricing are generalized by fair pricing, under appropriate assumptions.

\section{Risk-Neutral Pricing}

Two measures $P$ and $Q$ are said to be equivalent, if events having zero probability under one measure also have zero probability under the other. Let us discuss the special case where one additionally assumes the existence of an equivalent risk neutral martingale measure $Q$, which is chosen as pricing measure for the given market. Under such a prescribed financial market model with an equivalent risk neutral martingale measure, the Radon-Nikodym derivative process $\Lambda=\left\{\Lambda_{i}, i \in\right.$ $\{0,1, \ldots, n\}\}$ with

$$
\Lambda_{i}=\frac{S_{i}^{(0)}}{S_{0}^{(0)}} \frac{S_{0}^{(\underline{\pi})}}{S_{i}^{(\underline{\pi})}}=\frac{\hat{S}_{i}^{(0)}}{\hat{S}_{0}^{(0)}}
$$

for $i \in\{0,1, \ldots, n\}$, is then up to a factor, the benchmarked domestic savings account process $\hat{S}^{(0)}$, and thus an $(\underline{\mathcal{A}}, P)$-martingale, see Long (1990) or Platen (2002). Furthermore, the risk neutral measure $Q$ is characterized by the RadonNikodym derivative

$$
\left.\frac{d Q}{d P}\right|_{\mathcal{A}_{t_{n}}}=\Lambda_{n} .
$$

Let us describe how one obtains standard risk neutral contingent claim prices from the fair pricing formula (2.20). We consider again the contingent claim $H_{t_{n}}$, which 
is $\mathcal{A}_{t_{n}}$-measurable, expressed in units of domestic currency and paid at maturity $t_{n}$. Note that the claim $H_{t_{n}}$ can be contingent upon information obtained from the primary security account values $S_{i}^{(j)}$ for all $j \in\{0,1, \ldots, d\}$ and $i \in\{0,1, \ldots, n\}$, and also on additional information contained in $\mathcal{A}_{t_{n}}$.

To create the link to the risk neutral price, we note that the fair price $C_{H_{t_{n}}}\left(t_{i}\right)$, see (2.19) and (2.20), at time $t_{i}$ for the contingent claim $H_{t_{n}}$ is given by

$$
C_{H_{t_{n}}}\left(t_{i}\right)=E\left(\frac{S_{i}^{(\underline{\pi})}}{S_{n}^{(\underline{\pi})}} H_{t_{n}} \mid \mathcal{A}_{t_{i}}\right)
$$

for $t_{i} \leq t_{n}$. It thus follows by (2.21), (2.22) and (2.23) that

$$
\begin{aligned}
C_{H_{t_{n}}}\left(t_{i}\right) & =E\left(\frac{\Lambda_{n}}{\Lambda_{i}} \frac{S_{i}^{(0)}}{S_{n}^{(0)}} H_{t_{n}} \mid \mathcal{A}_{t_{i}}\right) \\
& =E\left(\left.\frac{d Q}{d P}\right|_{\mathcal{A}_{t_{n}}} \frac{S_{i}^{(0)}}{S_{n}^{(0)}} H_{t_{n}} \mid \mathcal{A}_{t_{i}}\right) \\
& =E^{Q}\left(\frac{S_{i}^{(0)}}{S_{n}^{(0)}} H_{t_{n}} \mid \mathcal{A}_{t_{i}}\right),
\end{aligned}
$$

where $E^{Q}(\cdot \mid \cdot)$ is the conditional expectation under the equivalent risk neutral martingale measure $Q$. Obviously, the above pricing formula (2.24) is the well-known risk neutral pricing formula, see (2.2). We have shown that it represents a special case of the fair pricing formula (2.20). The formula (2.24) is usually obtained by a Girsanov type change of measure, see Protter (1990). Note that if the financial market model does not have an equivalent risk neutral martingale measure, as is suggested by empirical evidence described in Platen (2004b), then the risk neutral pricing methodology is not available. However, fair pricing can still be performed.

\section{Generalized Actuarial Pricing}

In this subsection we consider a special case of fair pricing, where the payoff $H_{t_{n}}$ for a European contingent claim is independent of the GOP value $S_{n}^{(\pi)}$ at maturity $t_{n}$. To be more precise, we assume that $H_{t_{n}}$ is independent of $S_{n}^{(\underline{\pi})}$ conditionally on the information provided by $\mathcal{A}_{t_{i}}, i \in\{0,1, \ldots, n\}$. This can be assumed to be the case for the payoffs of weather derivatives because weather indices can be shown to be practically independent of the MSCI and thus the GOP. Exploiting this independence, we can write the conditional expectation in $(2.20)$ in the form

$$
\begin{aligned}
C_{H_{t_{n}}}\left(t_{i}\right) & =E\left(\frac{S_{i}^{(\underline{\pi})}}{S_{n}^{(\underline{\pi})}} H_{t_{n}} \mid \mathcal{A}_{t_{i}}\right) \\
& =E\left(\frac{S_{i}^{(\underline{\pi})}}{S_{n}^{(\underline{\pi})}} \mid \mathcal{A}_{t_{i}}\right) E\left(H_{t_{n}} \mid \mathcal{A}_{t_{i}}\right)
\end{aligned}
$$


for $i \in\{0,1, \ldots, n\}$. Note that the fair price $P\left(t_{i}, t_{n}\right)$ of a zero coupon bond at time $t_{i}$ with maturity $t_{n}$ is, by $(2.20)$, obtained as

$$
P\left(t_{i}, t_{n}\right)=E\left(\frac{S_{i}^{(\underline{\pi})}}{S_{n}^{(\underline{\pi})}} \mid \mathcal{A}_{t_{i}}\right),
$$

for $i \in\{0,1, \ldots, n\}$. We then arrive by $(2.25)$ and (2.26) at the generalized actuarial pricing formula

$$
C_{H_{t_{n}}}\left(t_{i}\right)=P\left(t_{i}, t_{n}\right) E\left(H_{t_{n}} \mid \mathcal{A}_{t_{i}}\right)
$$

This provides a theoretical derivation of equation (2.1) for the case when $H_{t_{n}}$ is independent of $S_{n}^{(\underline{\pi})}$. Note also that the interest rate term structure of the given model expressed via the zero coupon bond price (2.26) can be stochastic, which is often not the case for typically considered models in the actuarial literature.

The fair value $C_{H_{t_{n}}}\left(t_{i}\right)$ of an independent contingent claim $H_{t_{n}}$ at some time $t_{i}, i \in\{0,1, \ldots, n\}$, is by (2.27) the discounted value of the conditional expectation of the payoff value $H_{t_{n}}$, which refers to the well-known present value concept. Thus, the fair pricing formula (2.20) can be interpreted as a generalization of the actuarial pricing rule. It is worth noting that the knowledge of the values or dynamics of the GOP itself are not directly needed for the computation of the price in (2.27). Only the existence of the GOP is exploited in the above derivation. The impact of the GOP on the fair price is projected into the fair zero coupon bond price, which is readily available in a given market. This does not require the existence of an equivalent risk neutral martingale measure or the completeness of the market.

The fair pricing concept appears to be more general than the hedging approach for derivative pricing, first outlined by Black \& Scholes (1973) and Merton (1973). It is also consistent with the common belief in the Law of Large Numbers that usually motivates the actuarial or present value pricing approach. One can say that it has some similarity with the stochastic discount factor approach described in Cochrane (2001). Thus, it integrates several approaches. Once the fair price is known, in practice, a seller of a weather derivative can add a subjective liquidity premium as long as the market accepts that.

Using the fair pricing formula (2.20), or equivalently its incarnation as the generalized actuarial pricing formula (2.27), we will now determine fair prices for weather derivatives.

\subsection{Fair Pricing of Weather Derivatives}

\section{Weather Indices and Weather Derivative Payoffs}

A weather derivative is a contract that provides a payoff in response to a weather index level, which is in turn determined by weather phenomena. Daily average or 
daily maximum temperatures give examples of weather indices. Weather materially affects many industries, including agriculture, energy, tourism and retailing. The weather-related risks of one industry may offset those of another. In such a case, by swapping risks, two parties can safeguard their sales and profits from the particular set of weather conditions that would usually result in an uncertain profit and potential loss, see Kariya (2003). The stability of profits that arises from a weather derivative hedge is an attractive prospect for firms intent on securing earnings consistency.

An important aspect of weather derivatives is that they are financially settled. Since the underlying weather index is not a traded asset with a market determined price, a dollar multiplier $\lambda$ is used to convert the index into a dollar value.

To better explain the seasonal effects of temperature, we allow $t_{i}=t_{h, l}$ to represent the $h$ th day in the $l$ th year. Define $D_{t_{h, l}}, h \in\{1, \ldots, 365\}, l \in\{1,2, \ldots, n\}$ as the daily average temperature (DAT), measured as the arithmetic average of the maximum and minimum temperatures for the $h$ th day of the $l$ th year, disregarding leap years. The sum of the DATs in the $l$ th year represents the $l$ th cumulative annual temperature (CAT) $T_{t_{365, l}}, l \in\{1,2, \ldots, n\}$. That is,

$$
T_{t_{365, l}}=\sum_{h=1}^{365} D_{t_{h, l}},
$$

for $l \in\{1,2, \ldots, n\}$. With this we calculate the payoff of a European call option on the CAT with strike price $K$, maturity $t_{\bar{n}}$ and tick size $\lambda>0$, defined as a dollar value, to be

$$
H_{t_{\bar{n}}}=\lambda\left(T_{t_{\bar{n}}}-K\right)^{+},
$$

for $\bar{n} \in\{1,2, \ldots, n\}$. Here the maturity $t_{\bar{n}}$ of the option is not necessarily equivalent to the time $t_{n}$.

Temperature dependence, as defined by weather derivative instruments, can also be expressed in terms of degree days. A degree day is simply the difference between a fixed reference temperature $\kappa$ and the DAT. As a result, the weather derivative indices appear to be crude functions of the dynamics of daily and seasonal temperatures. However, they appear to serve most industries rather well. For example, there is a high correlation between natural gas demand and heating degree days (HDDs), which is of interest to gas companies and clients using gas for heating purposes.

A HDD is the maximum of zero and the difference between some reference temperature $\kappa$ and the DAT $D_{t_{h, l}}$ on the $h$ th day in the $l$ th year, $h \in\{1, \ldots, 365\}$, $l \in\{1,2, \ldots, n\}$. That is,

$$
H D D_{h, l}=\left(\kappa-D_{t_{h, l}}\right)^{+}
$$

In this paper, we use $\kappa=18^{\circ} \mathrm{C}$ as reference temperature. Alternatively, a cooling degree day $\mathrm{CDD}_{h, l}$ is the maximum of zero and the difference between the DAT 
$D_{t_{h, l}}$ at the $h$ th day in the $l$ th year $h \in\{1, \ldots, 365\}, l \in\{1,2, \ldots, n\}$, and the reference temperature $\kappa$. That is,

$$
C D D_{h, l}=\left(D_{t_{h, l}}-\kappa\right)^{+}
$$

It has been shown that there is a high correlation between CDDs and electricity usage, see Chau et al. (2000).

\section{Weather Derivative Contracts}

Weather derivatives are similar to equity options, except that the underlying asset is substituted by a weather index. For instance, one may choose a contract on the future CAT. Alternatively, one can use HDD and CDD contracts, which are written on the accumulation of HDDs or CDDs over a period of time. This period is typically a calendar month or an entire season. The index values are determined by the published temperature readings of a specified weather station reporting daily temperatures.

If the contract period is from $t_{\underline{h}, l}$ until $t_{\bar{h}, l}$, the accumulated HDDs or CDDs for that period are

$$
P_{t_{\underline{h}, l}, t_{\bar{h}, l}}^{H}=\sum_{h=\underline{h}}^{\bar{h}} H D D_{h, l}
$$

or

$$
P_{t_{\underline{h}, l}, t_{\bar{h}, l}}^{C}=\sum_{h=\underline{h}}^{\bar{h}} C D D_{h, l},
$$

respectively. The payoff $H_{t_{\bar{n}}}$ at time $t_{\bar{n}}=t_{h, l}$ of a European call option based on accumulated CDDs over this period is

$$
H_{t_{\bar{n}}}=\lambda\left(P_{t_{\underline{h}, l}, t_{\bar{h}, l}}^{C}-K\right)^{+},
$$

where $\lambda$ is the pre-defined tick size and $K$ is the strike. Other weather derivative contracts refer simply to maximum, minimum or average temperature indices.

We will restrict our discussion here to standard weather options. Similar arguments apply to weather derivative swaps, which are contracts where two parties exchange weather risks during an agreed period of time. Investor strategies using weather options are well documented, see for instance Leggio \& Lien (2002) and Kariya (2003).

As mentioned previously and will be illustrated below, weather indices can be regarded as independent of the GOP. Assuming that the weather index payoff $H_{t_{\bar{n}}}$ is independent of the GOP, the fair price $C_{H_{t_{\bar{n}}}}\left(t_{i}\right)$ at time $t_{i} \leq t_{\bar{n}}$ of a European option on a weather index is given by the generalized actuarial pricing formula (2.27). Thus, for instance, for the case of the CDD call option with payoff (2.34), we obtain by (2.27) the price

$$
C_{H_{t_{\bar{n}}}}\left(t_{i}\right)=P\left(t_{i}, t_{\bar{n}}\right) \lambda E\left(\left(P_{t_{\underline{h}, l}, t_{\bar{h}, l}}^{C}-K\right)^{+} \mid \mathcal{A}_{t_{i}}\right)
$$


at time $t_{i} \leq t_{\bar{n}}=t_{\bar{h}, l}$. In a similar way, it is possible also to price other weather derivatives, using the generalized actuarial pricing formula (2.27). The fair price, when expressed in units of the GOP, is a martingale. Thus, its expected future benchmarked value equals its present benchmarked value. In this sense, by taking expectation under the real world probability the fair price is the minimal price that can be sustained by a financial institution that issues weather derivatives and pools them. From a business perspective, the institution will need to charge some fee or liquidity premium to cover expenses, similar to market makers who live off the bid-ask spread. In reality, it can be expected that on top of fair prices, a liquidity or weather risk premium is charged that reflects the demand and supply of the respective products in the weather derivatives market.

\section{Data Analysis}

\subsection{Description of Data}

To illustrate the fair pricing concept under the benchmark approach, about 143 years of daily maximum and minimum temperatures were obtained for Sydney from the Commonwealth Bureau of Meteorology. The specific location for the observations is Observatory Hill, Sydney. The data is a complete set consisting of 52195 daily observations, from January 1859 to July 2002. Figures 1 and 2 show typical plots of the daily average temperature (DAT) over time. The DAT

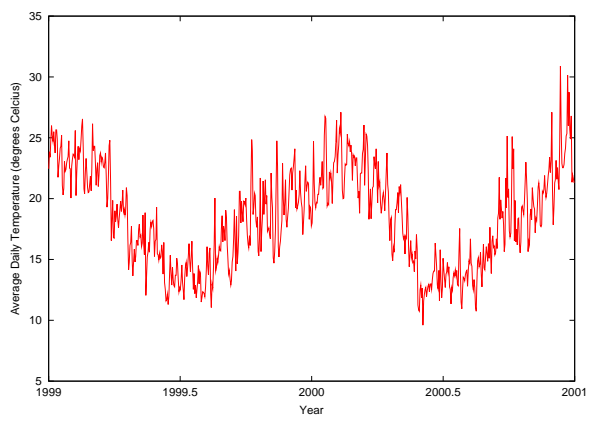

Figure 1: Daily average temperature, Sydney, Jan 1999-Dec 2000.

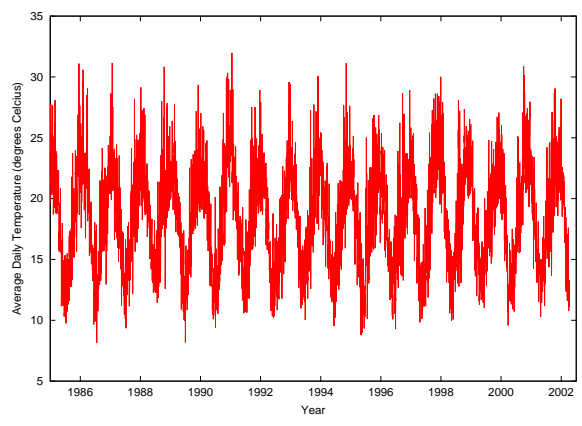

Figure 2: Daily average temperature, Sydney, Jan 1985 - Jul 2002.

for Sydney summer is about $23^{\circ} \mathrm{C}$, while the DAT during winter is around $13^{\circ} \mathrm{C}$. Figure 3 displays the cumulative annual temperature (CAT) for Sydney from 1859 to 2001. Each CAT is simply the average of the DATs of the respective year. It is apparent from the graphs that the raw CAT values are, on average, increasing over time. Minimum and maximum temperatures behave in a similar fashion. Due to global warming and urban heating conditions, the average temperature in Figure 3 appears to be curved upward. Predicting its near future average evolution may be realistic. Further to the trend that is visible in the 143 years of data, there exist long-term macro-cycles in temperature data, as can be obtained 


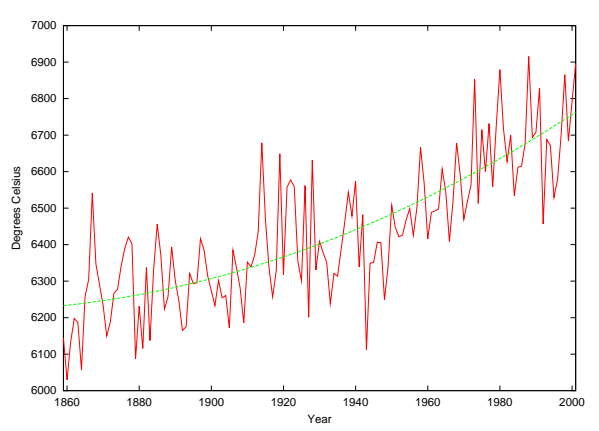

Figure 3: Cumulative annual temperature with trend, Sydney, 1859-2001.

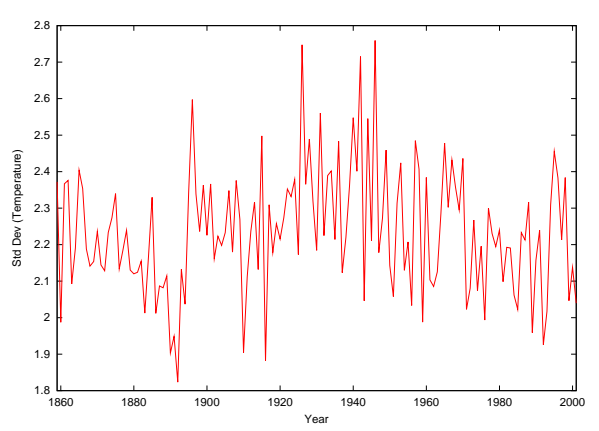

Figure 4: Standard deviation of $\mathrm{cu}-$ mulative annual temperatures, Sydney, 1859-2001.

from climatic change studies. We will assume that a certain systematic change in average temperature exists and we shall model some long term change. Figure 4 illustrates the standard deviation of CATs for Sydney. This value seems not to have changed significantly since 1859, even though CAT values have trended upwards by about 0.01 degrees Celsius per year over the past 143 years, see Figure 3 .

\subsection{Average}

Weather derivatives are flexible instruments that can be employed in both the long and short term. Agriculturalists may require financial risk protection over an entire year, while electricity distributors may require coverage for a number of days. In order to address different perspectives, the analysis of weather indices will be considered separately. Firstly, we will consider normalized CAT values. In assessing the yearly deviation of normalized CATs, the data could be detrended by simply subtracting the least squared trend.

To be more precise, we approximate the long term average with a second order polynomial curve, fitted to the data set for a weather index, to allow for the upward or downward trend where applicable. The average $A_{t_{h, l}}$, on day $h \in\{1, \ldots, 365\}$ in year $l \in\{1,2, \ldots, n\}$ is assumed to satisfy the equation

$$
A_{t_{h, l}}=\beta_{0} t_{h, l}^{2}+\beta_{1} t_{h, l}+\beta_{2}
$$

where $\beta_{g}$ are constant coefficients, $g \in\{0,1,2\}$. Table 1 shows the polynomial

\begin{tabular}{|c|c|c|c|c|}
\hline Index & $\beta_{0}$ & $\beta_{1}$ & $\beta_{2}$ & $\mu$ \\
\hline \hline Normalized cumulative annual temperature & 0.0191 & 0.8857 & 17.0978 & 18.3399 \\
& $(0.0064)$ & $(0.3109)$ & $(5.7528)$ & $(6.1707)$ \\
\hline
\end{tabular}

Table 1: Parameter estimates for the average of the normalized cumulative annual temperature.

coefficient estimates used to adjust the data series for the Sydney normalized CAT index along with the standard errors in parentheses, see Figure 3. The coefficient 
$\mu$ represents the normalized CAT mean, calculated from the average of the 143 years, as shown by the curved line in Figure 3. In Figure 5 we show the residuals of the Sydney normalized CAT index.

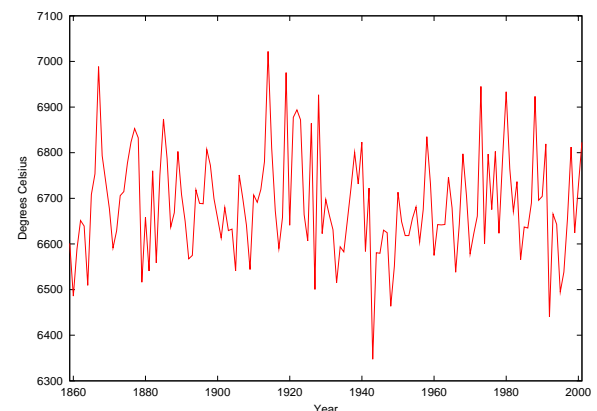

Figure 5: Residuals of Sydney normalized cumulative annual temperature, 1859-2001.

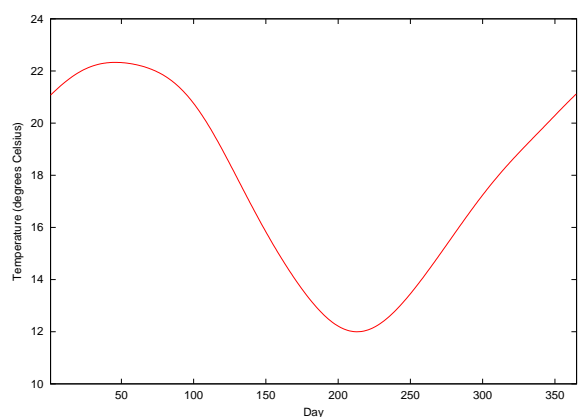

Figure 6: One cycle of seasonal pattern in daily average temperatures.

The linear component of the polynomial, characterized by $\beta_{1}$ and $\beta_{2}$, is important as it exerts far greater influence over the average than the second order component. The second order coefficient $\beta_{0}$ of the polynomial trendline is therefore mostly negligible.

\subsection{Seasonal Oscillating Average}

For several weather indices, systematic seasonal averages have to be taken into account. Let us appraise the average, for instance, in daily average temperatures (DATs) on a day by day basis. The seasonal cycle is removed by calculating each DAT's long-term average with a truncated Fourier series and then subtracting this average from each day's value to create a fluctuation record of residuals. Employing the above methodology for DATs requires an additive seasonal component $\xi_{t_{h, l}}$, in addition to the average given in (3.1), to provide a more accurate description of the index data, see Figures 1 and 2. The average thus becomes

$$
A_{t_{h, l}}=\beta_{0} t_{h, l}^{2}+\beta_{1} t_{h, l}+\beta_{2}+\xi_{t_{h, l}}
$$

for time $t_{h, l}, h \in\{1, \ldots, 365\}$ and $l \in\{1,2, \ldots, n\}$, where $\xi_{t_{h, l}}$ represents the seasonal oscillating average.

Suppose we model $\xi_{t_{h, l}}$ for $h \in\{1, \ldots, 365\}$ and $l \in\{1,2, \ldots, n\}$ by a truncated Fourier series to reflect the seasonal pattern in the form

$$
\xi_{t_{h, l}}=\sum_{k=1}^{N}\left[a_{k} \cos \left(2 \pi k \frac{\delta\left(t_{h, l}\right)}{L}\right)+b_{k} \sin \left(2 \pi k \frac{\delta\left(t_{h, l}\right)}{L}\right)\right],
$$

where $N$ is the number of frequencies considered. Furthermore, the number $L=$ 365 is the number of days per year, where we set $L=366$ for a leap year. The 
Fourier coefficients $a_{k}$ and $b_{k}$ are of the form

$$
a_{k}=\frac{2}{L} \sum_{h=1}^{L} f\left(t_{h, l}\right) \cos \left(\frac{k \pi \delta\left(t_{h, l}\right)}{L}\right)
$$

and

$$
b_{k}=\frac{2}{L} \sum_{h=1}^{L} f\left(t_{h, l}\right) \sin \left(\frac{k \pi \delta\left(t_{h, l}\right)}{L}\right)
$$

for $k \in\{1,2, \ldots, N\}$. Here, the periodic function $f\left(t_{h, l}\right)$ is approximated by the Fourier series $\xi_{t_{h, l}}$ and is defined for $t_{h, l}, h \in\{1, \ldots, 365\}$ and $l \in\{0,1, \ldots, n\}$. It is assumed that $f\left(t_{h, l}+L\right)=f\left(t_{h, l}\right)$, so that $f\left(t_{h, l}\right)$ has period $L$. The function $\delta\left(t_{h, l}\right), h \in\{1, \ldots, 365\}$ and $l \in\{0,1, \ldots, n\}$, is a repeating step function that counts each day of the year.

The truncated Fourier series was calibrated using standard Fourier analysis, see Ralston \& Rabinowitz (1978). Table 2 displays the obtained Fourier coefficients

\begin{tabular}{|c|c|c|c|c|}
\hline Parameter & Coefficient & & Parameter & Coefficient \\
\hline \hline$a_{1}$ & 4.7524 & & $b_{1}$ & 1.6963 \\
& $(0.8051)$ & & $(0.2925)$ \\
$a_{2}$ & -0.6134 & & $b_{2}$ & 0.1362 \\
& $(0.2191)$ & & $(0.0479)$ \\
$a_{3}$ & 0.0556 & $b_{3}$ & 0.0310 \\
& $(0.0254)$ & & $(0.0142)$ \\
$a_{4}$ & 0.0516 & $b_{4}$ & -0.0415 \\
& $(0.0280)$ & & $(0.0226)$ \\
$a_{5}$ & 0.0412 & & $b_{5}$ & 0.0232 \\
& $(0.0474)$ & & $(0.0266)$ \\
\hline
\end{tabular}

Table 2: Fourier coefficients for daily average temperature.

for DAT covering $N=5$ frequencies. This appears to be more than sufficient for practical purposes, since only the first two frequencies are really contributing. The standard errors are given in parentheses. In Figure 6 we show $\xi_{t_{h}}$ for the cycle of one year. Note that there is about a 50 day delay in the maximum and minimum temperatures of the cycle corresponding to the seasons, which is consistent with general experience.

Combining the estimated seasonal oscillating dynamics with the corresponding polynomial global upward trend in temperatures provides us with an approximation of the corresponding average $A_{t_{h, l}}$ underlying the data. Figure 7 illustrates a sample of the fitted values and residuals for Sydney DAT for the period 1993-1997. The residuals obtained from the entire data record exhibit no discernible seasonal variation and seem to be close to Gaussian, as illustrated in the relative frequency histogram of Figure 8. In particular, the estimated kurtosis is 2.976, which is very close to the Gaussian kurtosis of 3 .

There are longer term effects that do not allow themselves to be easily incorporated into reliable models. El Niño episodes are usually accompanied by sustained warm- 


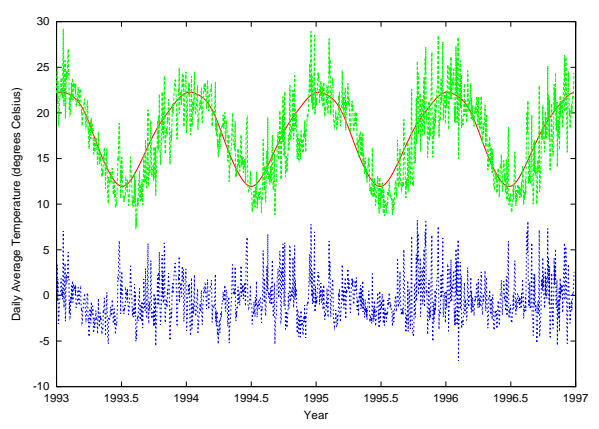

Figure 7: Daily average temperature fitted values and residuals, Sydney, 1993-1996.

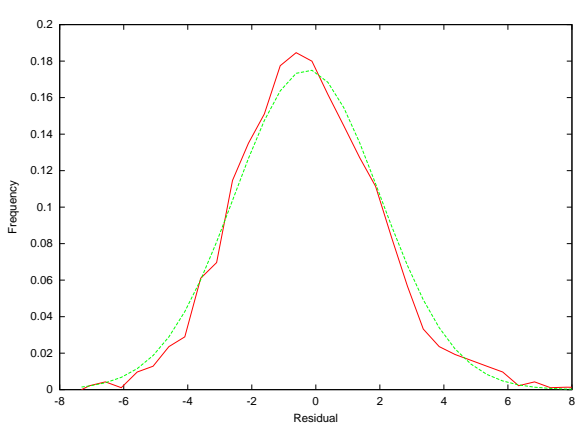

Figure 8: Relative frequency histogram of DAT residuals and a Gaussian density.

ing of the central and eastern tropical Pacific Ocean, a decrease in the strength of the Pacific Trade Winds and a reduction in rainfall over eastern and northern Australia. La Niña episodes are associated with stronger Pacific Trade Winds and warmer sea temperatures to the north of Australia. These two effects can significantly alter observed temperature patterns for several consecutive years in Sydney. Global effects that occur over the long term, such as these, can attenuate temperature modeling errors. A switching regime or similar mechanism could be incorporated into the modeling of the average of weather indices to broadly account for hotter or colder average global temperatures due to identified patterns such as the El Niño. However, this needs a much longer data set than is used here.

\section{Pricing Methods}

\subsection{Fair Weather Derivative Pricing}

As mentioned previously, traditional financial derivative pricing utilizes hedging arguments which require the underlying asset to be tradable. Underlying weather indices or futures contracts on these indices are, so far, rarely liquidly traded. Therefore, in practice, the hedging argument cannot be directly applied to price weather contracts. The lack of a trading history for weather derivatives also precludes a correlation analysis with other financial instruments. However, we can reasonably assume independence of weather indices from globally diversified world stock accumulation indices. The growth optimal portfolio (GOP) can be interpreted according to Platen (2004b), for instance, as being approximated by the MSCI. We show in Figure 9 the logarithms of the daily MSCI Growth World Index and of the Sydney DAT from 1975 to 2002. The correlation coefficient of the increments of the two series is about 0.00134, suggesting that no significant correlation is detectable. This indicates that weather fluctuations are unlikely to be affected by those of the GOP or vice versa. 


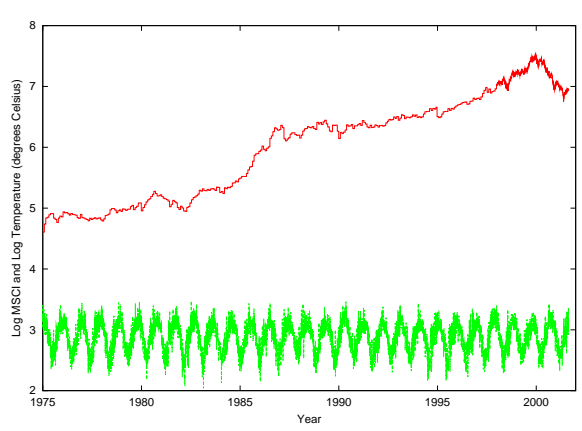

Figure 9: Log of the MSCI World Index and Sydney daily average temperature, 1975-2002.

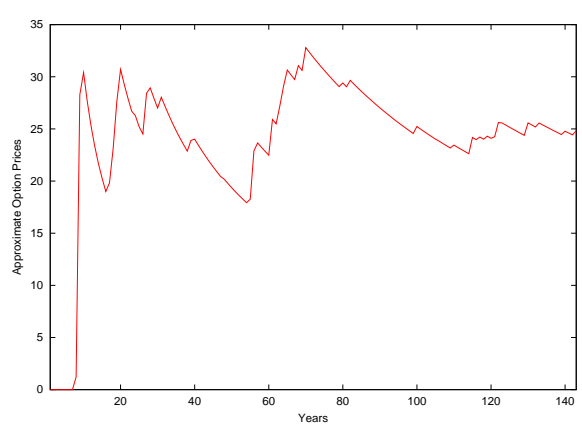

Figure 10: Convergence of approximate fair option price for increasing data used in the HFP method.

As already shown in Section 2.2 the assumption of independence between the GOP and the underlying weather index leads to the generalized actuarial pricing formula (2.27). This formula only requires the computation of the real world expectation $E\left(H_{t_{\bar{n}}} \mid \mathcal{A}_{t_{i}}\right)$ of the contract payout $H_{t_{\bar{n}}}$. The fair value of a contingent claim $C_{H_{t_{\bar{n}}}}\left(t_{i}\right)$, at some time $t_{i} \leq t_{\bar{n}}$, is then simply the above expectation multiplied by the zero coupon bond price $P\left(t_{i}, t_{\bar{n}}\right)$, which acts as a discounting factor. This is a simple but powerful result, which makes it easy to price weather derivatives efficiently, if a sufficient temperature record is available.

Therefore, as an illustration, the fair value of a CAT option, calculated for a specific location, will be the expected positive part of the difference between the value of the CAT index and the nominated strike value, discounted at the prevailing discount rate. The only task that remains, in practice, is to calculate the expected value of the payoff $E\left(H_{t_{\bar{n}}} \mid \mathcal{A}_{t_{i}}\right)$.

\subsection{Historical Fair Pricing}

In this subsection we introduce what we call the historical fair pricing (HFP) method. This procedure is extremely simple and similar to an historical simulation or burn analysis, see Kariya (2003), which is sometimes used for pricing insurance contracts. We will demonstrate this method by using the residuals of the available CAT index observations to approximate the required expectation by a corresponding average. The method is supported by the Law of Large Numbers, if one assumes sufficient historical data and independence of the observed CATs.

Let $\hat{\sigma}$ represent the estimated standard deviation of all CATs in our data set

$$
\hat{\sigma}=\sqrt{\frac{1}{142} \sum_{t_{l}=1}^{143}\left(T_{t_{l}}-\hat{\mu}\right)^{2}},
$$


where $\hat{\mu}$ is the estimated average of the CAT. Suppose we set a strike

$$
K=\frac{1}{143} \sum_{l=1}^{143} T_{t_{l}}+\frac{\hat{\sigma}}{2}
$$

at half of a standard deviation $\frac{\hat{\sigma}}{2}$ off the estimated mean CAT value $\hat{\mu}$. In the absence of observed strike values in the market, we use the above strike for illustrative purposes. We now calculate the expected option payoff $E\left(H_{t_{\bar{n}}} \mid \mathcal{A}_{t_{i}}\right)$ for $t_{i} \leq t_{\bar{n}}$, based on the 143 adjusted residuals that our data set provides. For the pricing of this contract, we use the entire record of CAT residuals to estimate the expected payoff. We make the assumption that the residuals of the CATs for each year are identically distributed and independent. This provides reasonable results, since the adjusted fluctuations over the entire period are relatively stable. We calculate the adjusted payoffs for all years in the historical record and average them to estimate the conditional expectation of the payoff. Discounting the estimated expected value of the weather derivative payoff with the zero coupon bond $P\left(t_{i}, t_{\bar{n}}\right)$ provides a proxy for the fair price at time $t_{i}$ by $(2.27)$. Fair European option values were calculated using the above settings, along with a tick size $\lambda=\$ 1$, defined previously in (2.29). Table 3 presents the results of the HFP method for European call and put options with a maturity of one year and strike $K$, see (4.2). As we see in Table 4, the estimated standard deviation of the CAT residuals is $\hat{\sigma}=115.6076$ and the estimated mean is $\hat{\mu}=6686.4314$.

\begin{tabular}{|c|c|c|c|}
\hline Option Type & Strike $K$ & Option Value $C_{H_{t_{\bar{n}}}}\left(t_{i}\right)$ & Gaussian Model \\
\hline \hline CAT Call & 6744 & 24.79 & 24.55 \\
CAT Put & 6629 & 19.34 & 19.68 \\
\hline
\end{tabular}

Table 3: CAT option values.

Figure 10 illustrates the convergence of approximate option prices as the data used in the HFP method increases. It seems that at least 110 years of data is required for the HFP method to achieve reasonable convergence. Option values calculated using only short term data appear to be unreliable, since prices vary significantly from the price calculated using all historical data. However, the advantage of the HFP method is that it is simple and does not use any assumption on the distribution of the residuals.

The process of applying the HFP method for pricing weather derivatives is not limited to a single maturity payoff. It can accommodate the complexity of many weather derivative instruments. We emphasize that it is important to work with residuals and not with raw data, in order to correctly incorporate the impact of long term trends and oscillations.

\subsection{Fair Pricing Under a Distributional Model}

Let us now describe the fair pricing of weather derivatives using a model for the distribution of the weather index value at maturity. The value of a CAT call 
option calculated for a specific location equals, according to (2.27) and (2.29), the expected value of the positive part of the difference between the value of the CAT and the nominated strike value, discounted back to the settlement date.

We will be concerned with the fair value satisfying the generalized actuarial pricing formula (2.27), which refers to independence of weather risk from the GOP. This allows us to follow a purely statistical approach, in which historical records of the observed physical weather index process are the source for the calibration of the model. In the simplest case, such a model could be described by a given parametric distribution function for the value of a weather index at a given time. This leads to the estimation of the associated parameters. Under a Gaussian distributional assumption, these would be the mean $\mu$ and the standard deviation $\sigma$. The expected payoff is then a consequence of the model.

Let us indicate why the Gaussian assumption is rather natural for certain weather indices, such as CAT. Suppose that $X_{1}, X_{2}, \ldots, X_{n}$ forms a sequence of independent and identically distributed random variables, for example DATs, with finite mean $\mu$ and finite variance $\sigma^{2}>0$. Let us introduce the sample average

$$
\hat{\mu}_{X^{(m)}}=\frac{1}{m} \sum_{i=1}^{m} X_{i}
$$

and the sample variance

$$
\hat{\sigma}_{X^{(m)}}^{2}=\frac{1}{m-1} \sum_{i=1}^{m}\left(X_{i}-\hat{\mu}_{X^{(m)}}\right)^{2} .
$$

The sample will have expected value $E\left(\hat{\mu}_{X^{(m)}}\right)=\mu_{X}$. The Law of Large Numbers states that $\hat{\mu}_{X^{(m)}} \rightarrow \mu_{X}$ in probability as $m \rightarrow \infty$. Let us introduce the normalized sample average

$$
Z_{m}=\frac{\sqrt{m}\left(\hat{\mu}_{X^{(m)}}-\mu_{X}\right)}{\hat{\sigma}_{X^{(m)}}} .
$$

Then, by the Central Limit Theorem, $Z_{m}$ converges in distribution to a standard normal random variable $Z \sim N(0,1)$ as $m \rightarrow \infty$. The distribution of $Z_{m}$ tends to a normal one, regardless of the distribution of the elements of the sequence $X_{1}, \ldots, X_{m}$ from which the random sample is drawn.

Table 4 outlines the descriptive statistics for CATs, obtained from raw index data and also from adjusted data when the changing average is taken into account, as described in Section 3. The values $\hat{\mu}$ and $\hat{\sigma}$ represent the estimated CAT index mean and deviation, respectively. Below both $\hat{\mu}$ and $\hat{\sigma}$ we show, in parentheses, the respective daily average values.

The quantity $\mathcal{X}^{2}$ GoF is the Chi Squared Goodness of Fit test statistic. At the $5 \%$ significance level, its critical value is 21.02607. Both the chi-squared goodnessof-fit and Jarque-Bera tests for normality in Table 4 imply that the CATs are indeed close to normal. The Jarque-Bera statistic jointly tests the third and fourth 


\begin{tabular}{|c|c|c|c|c|c|c|}
\hline Type & $\hat{\mu}$ & $\hat{\sigma}$ & Skew & Kurt & $\mathcal{X}^{2}$ GoF & Jarque-Bera \\
\hline \hline Unadjusted & 6435.98 & 191.1972 & 0.3935 & 2.6924 & 18.3239 & 4.6868 \\
Average Temp & $\left(17.63^{\circ} \mathrm{C}\right)$ & $\left(10.01^{\circ} \mathrm{C}\right)$ & & & & \\
Adjusted & 6686.4314 & 115.6076 & 0.3767 & 3.4679 & 18.0366 & 4.2846 \\
Average Temp & $\left(18.32^{\circ} \mathrm{C}\right)$ & $\left(6.05^{\circ} \mathrm{C}\right)$ & & & & \\
\hline
\end{tabular}

Table 4: Descriptive statistics for cumulative annual temperatures.

moments of a distribution against a chi-squared distribution with two degrees of freedom. This detects the presence of either skewness or kurtosis in the given distribution. The level of significance is chosen to be $5 \%$, which corresponds to 5.9915 as the critical level for the test statistic.

On the evidence above, the Gaussian assumption cannot be rejected at the given level of significance for both adjusted and unadjusted Sydney indices. Figures 11 and 12 are QQ-plots for Sydney CATs and the CDD index. They show fairly

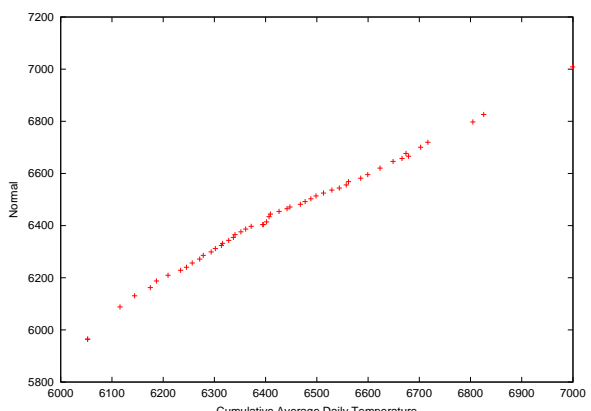

Figure 11: QQ-plot of cumulative annual temperature for Sydney, 18592002 .

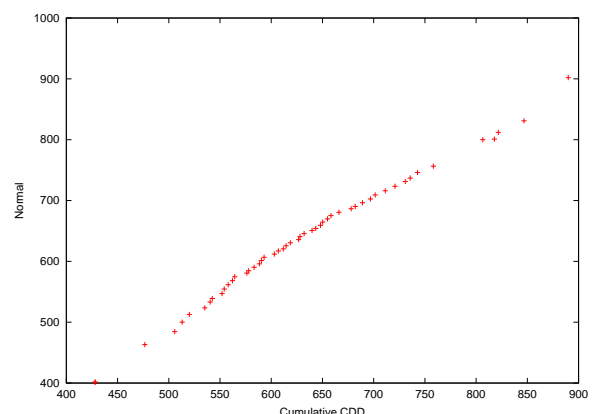

Figure 12: QQ-plot of cumulative CDD index values for Sydney, 18592002.

linear patterns, in particular in the tails, as is characteristic of a Gaussian sample. The relative frequency histogram of DAT residuals also indicates the Gaussian property.

The payoff of a European call weather option, with strike $K$ and maturity $t_{\bar{n}}$, on a Gaussian distributed weather index, with mean $\mu$ and variance $\sigma^{2}$, yields a fair derivative value

$$
C_{H_{t_{\bar{n}}}}\left(t_{i}\right)=P\left(t_{i}, t_{\bar{n}}\right) E\left(H_{t_{\bar{n}}}\right)=P\left(t_{i}, t_{\bar{n}}\right)\left[(\mu-K) N\left(\frac{\mu-K}{\sigma}\right)+\sigma N^{\prime}\left(\frac{K-\mu}{\sigma}\right)\right],
$$

by (2.26) and a straightforward calculation. Here $N(x)$ is the cumulative standard normal distribution function and $\bar{n}$ represents the number of the day when the option expires. It is clear that (4.6) is different from the Black-Scholes formula, because the underlying is normal and not lognormal. Fair prices for options on CATs under the above Gaussian model are comparable to those obtained using the HFP method, as is shown in Table 3.

The advantage of fair pricing under a distributional model is that one obtains a 
simple expression for the option price in terms of the parameters. On the other hand, one needs to validate the applicability of the model and calibrate it. If enough data is available, then fair pricing via a distributional model should provide similar results to the HFP method.

\subsection{Wine Producer Example}

To provide a reasonably realistic and still simple example in an area where weather is crucial, consider a viticulturist in the Hunter Valley, a popular vineyard province north of Sydney. The harvest of grapes usually occurs in early March. The climatic conditions during the two months prior to the harvest have the greatest impact on the quality of the fruit. Temperature is known to be a critical determinant of plant growth and fruit flavor quality. The optimal climatic conditions vary with grape variety. Happ (1999) claims that the optimal temperatures for flavor production and conservation are between $16^{\circ} \mathrm{C}$ and $22^{\circ} \mathrm{C}$, however, fruit maturation and acid retention is influenced markedly above $25^{\circ} \mathrm{C}$.

Although it is less than ideal to rely upon an average temperature to describe the day's thermal character, it serves as a reasonable proxy for obtaining information on the potential quality of a grape harvest. Harvest usually occurs in early March, so the two months prior to the harvest represent a 59-day window in which temperature has the greatest impact on fruit quality. In order to hedge against potential loss, a viticulturist can purchase a weather derivative based on the daily average temperature (DAT). In order to obtain fair prices for such contracts, we use the methodology outlined in Section 3, with reference to the generalized actuarial pricing formula (2.27).

The temperatures in the Hunter Valley region are highly correlated with temperatures in Sydney. The correlation coefficient for DAT between the two locations is 0.9967 . Therefore, we can use the temperature series for Sydney as a proxy for temperatures in the Hunter Valley.

Consider a wine producer who wishes to purchase a call option on an appropriate weather index. The call option will be based on cooling degree days (CDDs). The length of the contract will be two months, starting on 1 January and finishing on 28 February. Let the tick size $\lambda$ defined in (2.35) be $\$ 1$.

The reference temperature $\kappa$ for a CDD is $18^{\circ} \mathrm{C}$, as defined in (2.33). The CDDs are accumulated throughout the two months for the contract, as shown in (2.33). A strike value $K=308$ is chosen for the resulting two-month CDD index. This value represents well the historical evidence of crop damage from extreme temperatures.

The raw data is adjusted to incorporate the gradual global rise in temperature over the past 143 years. Using a polynomial approximation of the type (3.1) to fit historical values for two-month accumulated CDDs results in the parameter estimates given in Table 5, where estimates that have significance at a $5 \%$ level 


\begin{tabular}{|c|c|c|c|c|}
\hline Index & $\beta_{0}$ & $\beta_{1}$ & $\beta_{2}$ & Standard Error \\
\hline \hline Accumulated two-month CDD & $0.0036^{*}$ & -0.010495 & $224.6356^{*}$ & 36.5472 \\
& $(0.0012)$ & $(0.0037)$ & $(71.6349)$ & \\
\hline
\end{tabular}

Table 5: Parameter estimates for average accumulated two-month CDDs.

are indicated by * and we show in parentheses the standard error around the polynomial approximation. These values, when applied to the raw data, result in the adjusted accumulated two-month CDDs shown in Figure 13, using the procedure outlined in Section 3.

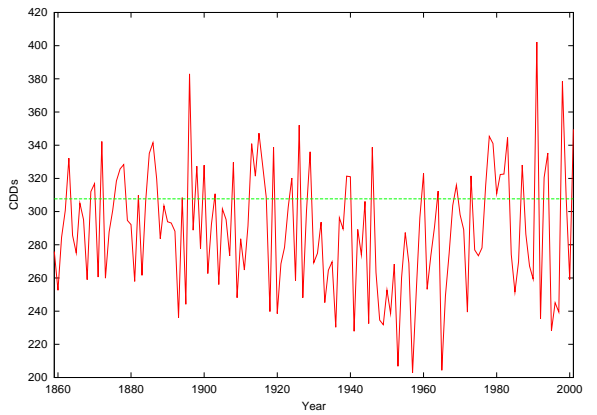

Figure 13: Adjusted daily average temperature CDD index values for Jan/Feb Sydney, 1859-2001.

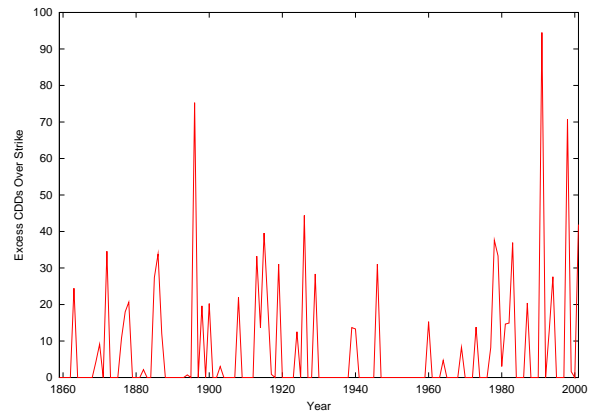

Figure 14: Historical payoffs for average daily temperature CDD index values for Sydney, 1859-2001.

The adjusted historical payoffs used for the HFP method for the European call option contract with strike $K=308$ are illustrated in Figure 14. In order to obtain the fair price $C_{H_{t_{\bar{n}}}}\left(t_{i}\right)$ for this contract, the formula (2.27) can be used. For the given contract, the expected payoff at maturity is estimated by applying the HFP method, which yields the value

$$
E\left(\left(P_{t_{\underline{h}, l}, t_{\bar{h}, l}}^{C}-K\right)^{+} \mid \mathcal{A}_{t_{i}}\right) \approx 7.5563 .
$$

A deterministic interest rate of $5 \%$ results then in an estimated fair value for the option of

$$
C_{H_{t_{\bar{n}}}}\left(t_{i}\right) \approx 7.4955 \text {. }
$$

A financial institution that sells such fair weather derivatives in a geographically and temporally diversified manner can pool these contracts and, on average, make a profit if it charges some additional fee or liquidity premium on top of the fair value. For buyers and sellers the fair price is always an important reference value. If futures on weather indices and weather derivatives become liquidly traded, then a financial institution can, in principle, hedge such contracts. Also, in this case, the institution can still profit from fees similar to bid and ask spreads in other markets. 


\section{Conclusion}

An important general link between financial and actuarial pricing methodologies has been described and exploited for weather derivatives, using the concept of fair pricing under the benchmark approach. Fair values for weather derivatives are estimated by using the residuals of historical temperature data and a Gaussian model. In both cases we correct for seasonal and long term trends. The emergence of new weather indices and instruments will further add to the liquidity and competitiveness of the weather derivatives market. This will benefit many industries which at present, cannot escape exposure to large weather fluctuations. It will also lead to the possibility of hedging weather derivatives. We have argued in this paper that in a liquid, competitive weather derivative market, zero risk premia can be expected to exist, due to the diversifiability of weather risk. However, in the present still emerging weather derivative market, certain liquidity premia or fees are likely to be charged.

\section{References}

Bajeux-Besnainou, I. \& R. Portait (1997). The numeraire portfolio: A new perspective on financial theory. The European Journal of Finance 3, 291309.

Becherer, D. (2001). The numeraire portfolio for unbounded semimartingales. Finance Stoch. 5, 327-341.

Black, F. \& M. Scholes (1973). The pricing of options and corporate liabilities. J. Political Economy 81, 637-659.

Borch, K. H. (1968). The Economics of Uncertainty. Princeton University Press.

Bühlmann, H. \& E. Platen (2003). A discrete time benchmark approach for insurance and finance. ASTIN Bulletin 33(2), 153-172.

Cao, M. \& J. Wei (2001). Equilibrium valuation of weather derivatives. Working Paper, York University and University of Toronto.

Chau, T., S. Makita, G. Riopel, \& T. Wang (2000). Modelling and hedging electricity generation profits. Financial Engineering Proseminar, MIT Sloan School of Management.

Cochrane, J. H. (2001). Asset Pricing. Princeton University Press.

Delbaen, F. \& W. Schachermayer (1994). A general version of the fundamental theorem of asset pricing. Math. Ann. 300, 463-520.

Fama, E. F. \& J. D. MacBeth (1974). Long-term growth in a short-term market. J. Finance 29, 857-885.

Goll, T. \& J. Kallsen (2003). A complete explicit solution to the log-optimal portfolio problem. Adv. in Appl. Probab. 13(2), 774-799. 
Happ, E. (1999). Indices for exploring the relationship between temperature and grape and wine flavour. Wine Industry Journal 14(4), 1-5.

Heath, D., E. Platen, \& M. Schweizer (2001). A comparison of two quadratic approaches to hedging in incomplete markets. Math. Finance 11(4), 385413.

Hofmann, N., E. Platen, \& M. Schweizer (1992). Option pricing under incompleteness and stochastic volatility. Math. Finance 2(3), 153-187.

Kariya, T. (2003). Weather risk swap valuation. Proceedings JAFFEE International Conference, 466-481. March 15-16, 2003, Tokyo, Hitotsubashi University.

Kelly, J. R. (1956). A new interpretation of information rate. Bell Syst. Techn. J. 35, 917-926.

Korn, R. \& M. Schäl (1999). On value preserving and growth-optimal portfolios. Math. Methods Oper. Res. 50(2), 189-218.

Leggio, K. B. \& D. Lien (2002). Hedging gas bills with weather derivatives. J. Economics \& Finance 26(1), 88-100.

Long, J. B. (1990). The numeraire portfolio. J. Financial Economics 26, 29-69.

McIntyre, R. \& S. Doherty (1999). Weather risk - an example from the UK. Energy and Power Risk Management. June.

Merton, R. C. (1973). Theory of rational option pricing. Bell J. Econ. Management Sci. 4, 141-183.

Platen, E. (2002). Arbitrage in continuous complete markets. Adv. in Appl. Probab. 34(3), 540-558.

Platen, E. (2004a). A benchmark framework for risk management. In Stochastic Processes and Applications to Mathematical Finance, pp. 305-335. Proceedings of the Ritsumeikan Intern. Symposium: World Scientific.

Platen, E. (2004b). Modeling the volatility and expected value of a diversified world index. Int. J. Theor. Appl. Finance 7(4), 511-529.

Platen, E. (2004c). Pricing and hedging for incomplete jump diffusion benchmark models. In Mathematics of Finance, Volume 351 of Contemporary Mathematics, pp. 287-301. American Mathematical Society.

Protter, P. (1990). Stochastic Integration and Differential Equations. Springer.

Ralston, A. \& P. Rabinowitz (1978). A First Course in Numerical Analysis (2nd ed.). New York: Dover Publications.

Samuelson, P. A. (1971). The fallacy of maximising the geometric mean in long sequences of investing or gambling. Proceedings of the National Academy of Science, 2493-2496. 